[23] S. Bhasin, N. Sharma, P. Patre, and W. E. Dixon, "Asymptotic tracking by a reinforcement learning-based adaptive critic controller," J. Control Theory and App., vol. 9, no. 3, pp. 400-409, 2011.

[24] C. Makkar, G. Hu, W. G. Sawyer, and W. E. Dixon, "Lyapunov-based tracking control in the presence of uncertain nonlinear parameterizable friction," IEEE Trans. Autom. Control, vol. 52, pp. 1988-1994, 2007

[25] W. E. Dixon, M. S. de Queiroz, D. M. Dawson, and F. Zhang, "Tracking control of robot manipulators with bounded torque inputs," Robotica, vol. 17, pp. 121-129, 1999.

[26] P. M. Patre, W. Mackunis, C. Makkar, and W. E. Dixon, "Asymptotic tracking for systems with structured and unstructured uncertainties," IEEE Trans. Control Syst. Technol., vol. 16, pp. 373-379, 2008.

[27] P. M. Patre, W. MacKunis, K. Kaiser, and W. E. Dixon, "Asymptotic tracking for uncertain dynamic systems via a multilayer neural network feedforward and RISE feedback control structure," IEEE Trans. Autom, Control, vol. 53, no. 9, pp. 2180-2185, Sep. 2008.

[28] M. de Queiroz, J. Hu, D. Dawson, T. Burg, and S. Donepudi, “Adaptive position/force control of robot manipulators without velocity measurements: Theory and experimentation," IEEE Trans. Syst. Man Cybern., vol. 27-B, no. 5, pp. 796-809, 1997.

[29] A. F. Filippov, Differential Equations With Discontinuous Right-Hand Sides. Norwell, MA: Kluwer, 1988.

[30] J. P. Aubin and H. Frankowska, Set-Valued Analysis. Basel, Switzerland: Birkhäuser, 2008.

[31] D. Shevitz and B. Paden, "Lyapunov stability theory of nonsmooth systems," IEEE Trans. Autom. Control, vol. 39, no. 9, pp. 1910-1914, Sep. 1994.

[32] B. Paden and S. Sastry, "A calculus for computing Filippov's differential inclusion with application to the variable structure control of robot manipulators," IEEE Trans. Circuits Syst., vol. CAS-34, no. 1, pp. 73-82, 1987.

[33] F. H. Clarke, Optimization and Nonsmooth Analysis. Philadelphia, PA: SIAM, 1990.

[34] R. Leine and N. van de Wouw, "Non-smooth dynamical systems," in Stability and Convergence of Mechanical Systems With Unilateral Constraints, ser. Lecture Notes in Applied and Computational Mechanics. Berlin/Heidelberg: Springer, 2008, vol. 36, pp. 59-77.

[35] R. Kamalapurkar, J. Klotz, R. Downey, and W. E. Dixon, 2013 , Supporting Lemmas for RISE-Based Control Methods arXiv:1306. 3432.

[36] N. Fischer, R. Kamalapurkar, and W. E. Dixon, "LaSalle-Yoshizawa corollaries for nonsmooth systems," IEEE Trans. Automat. Control, vol. 58, no. 9, pp. 2333-2338, 2013.

[37] K. S. Walgama, S. Ronnback, and J. Sternby, "Generalization of conditioning technique for anti-windup compensators," Proc. IEE Control Theory Appl., vol. 139, no. 2, pp. 109-118, 1992.

\section{Sensitivity Reduction by Stable Controllers for MIMO Infinite Dimensional Systems via the Tangential Nevanlinna-Pick Interpolation}

\author{
Masashi Wakaiki, Student Member, IEEE, \\ Yutaka Yamamoto, Fellow, IEEE, and \\ Hitay Özbay, Senior Member, IEEE
}

\begin{abstract}
We study the problem of finding a stable stabilizing controller that satisfies a desired sensitivity level for an MIMO infinite dimensional system. The systems we consider have finitely many simple transmission zeros in $\overline{\mathbb{C}}_{+}$, but they are allowed to possess infinitely many poles in $\mathbb{C}_{+}$. We compute both upper and lower bounds of the minimum sensitivity achievable by a stable controller via the tangential Nevanlinna-Pick interpolation. We also obtain stable controllers attaining such an upper bound. To illustrate the results, we discuss a repetitive control system as an application of the proposed method.
\end{abstract}

Index Terms $-\mathcal{H}^{\infty}$ control, infinite dimensional systems, strong stabilization, tangential interpolation.

\section{INTRODUCTION}

The purpose of this note is to find stable controllers achieving a desired sensitivity level for MIMO infinite dimensional systems. Let us first note that even for stable plants, $\mathcal{H}^{\infty}$ optimization may produce unstable controllers. However, such controllers have difficulties with robustness and hardware implementation. Indeed, an unstable controller can lead to instability of the closed-loop system if a component such as a sensor or an actuator fails [1] or saturates [2]. See also [3]-[5] for theoretical and practical significance of stabilization by a stable controller. Applications of stable $\mathcal{H}^{\infty}$ controllers can be found in flexible structures [6], DC servo motors [7], data-communication networks [8], etc.

For SISO infinite dimensional systems, the Nevanlinna-Pick interpolation [9], [10] enables us to design stable controllers providing the minimum sensitivity [11] or robust stability [12]. The point of this approach is that a stable controller stabilizes the plant if and only if a unit element in $\mathcal{H}^{\infty}$ satisfies certain interpolation conditions at the unstable zeros of the plant. On the other hand, for MIMO infinite dimensional systems, the stable $\mathcal{H}^{\infty}$ controller design problem is still largely open. This is due to the difficulty of multivariable zeros.

We have studied sensitivity reduction by a stable controller for MIMO systems with infinitely many unstable poles in [13]. We have shown there that the matrix-valued Nevanlinna-Pick interpolation [3], [14] gives a sufficient condition and also a necessary condition for this problem. However the results in [13] are subject to the rather stringent assumption that all unstable zeros of the plant be blocking

Manuscript received May 08, 2013; revised August 23, 2013 and September 11, 2013; accepted September 29, 2013. Date of publication October 17, 2013; date of current version March 20,2014. This paper was presented in part at the 51st IEEE Conference on Decision and Control. Recommended by Associate Editor L. Mirkin.

M. Wakaiki and Y. Yamamoto are with the Department of Applied Analysis and Complex Dynamical Systems, Graduate School of Informatics, Kyoto University, Kyoto 606-8501, Japan (e-mail: wakaiki@acs.i.kyoto-u.ac.jp; yy@i. kyoto-u.ac.jp).

H. Özbay is with the Department of Electrical and Electronics Engineering, Bilkent University, Bilkent, Ankara TR-06800,Turkey (e-mail: hitay@bilkent. edu.tr).

Color versions of one or more of the figures in this paper are available online at $\mathrm{http}: / /$ ieeexplore.ieee.org.

Digital Object Identifier 10.1109/TAC.2013.2285788 


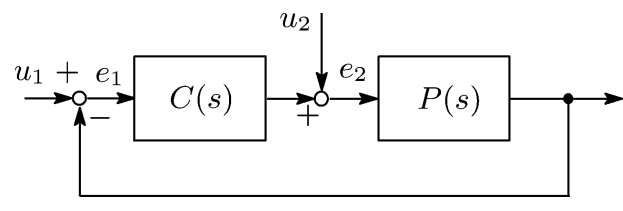

Fig. 1. Closed-loop system.

zeros, which can be treated in a way similar to that of zeros of SISO systems. Then we encounter the following question: Can we still solve this problem for MIMO infinite dimensional systems with unstable "transmission" zeros by the Nevanlinna-Pick interpolation? If so, what kind of interpolation do we need?

In this technical note, we consider MIMO plants with equal numbers of inputs and outputs only. However they are allowed to possess finitely many simple transmission zeros in $\overline{\mathbb{C}}_{+}$and infinitely many poles in $\mathbb{C}_{+}$. With the aid of Cramer's rule, we first show that stabilization by a stable controller is equivalent to tangential interpolation by a unimodular matrix. Next we obtain both a sufficient condition and a necessary condition for sensitivity reduction by a stable controller, using the tangential Nevanlinna-Pick interpolation with boundary conditions. This interpolation problem is solvable if and only if the Pick matrix consisting of the interpolation data at the interior points of $\mathbb{C}_{+}$is positive definite [10]. Thus we can compute upper and lower bounds of the minimum sensitivity by iterative calculations of the associated Pick matrices. We also design stable controllers achieving such an upper bound.

The technical note is organized as follows: In Section II, we give the problem formulation of sensitivity reduction by a stable controller. In Section III, we first transform this problem to a tangential interpolation by a unimodular matrix in $\mathbf{M}\left(\mathcal{H}^{\infty}\right)$ whose norm is less than one. Next we obtain both upper and lower bounds of the minimum sensitivity achievable by a stable controller. Section IV presents two numerical examples and the second example illustrates application to a repetitive control system. Concluding remarks are made in Section V.

\section{Notation and Definitions}

Let $\mathbb{C}_{+}, \overline{\mathbb{C}}_{+}$, and $j \mathbb{R}$ denote the open right half-plane $\{s \in \mathbb{C} \mid \operatorname{Re} s>$ $0\}$, the closed right half-plane $\{s \in \mathbb{C} \mid \operatorname{Re} s \geq 0\}$, and the imaginary axis $\{j \omega \mid \omega \in \mathbb{R}\}$, respectively. We define $\mathcal{H}^{\infty}$ to be the collection of all analytic and bounded functions on $\mathbb{C}_{+} \cdot \mathcal{R} \mathcal{H}^{\infty}$ denotes the subset of $\mathcal{H}^{\infty}$ consisting of rational functions with real coefficients. We denote by $\mathcal{F}^{\infty}$ the field of fractions of $\mathcal{H}^{\infty}$.

For the commutative ring $R, \mathbf{M}(R)$ denotes the set of matrices with entries in $R$, of whatever order. When it is necessary to display explicitly the order of a matrix, we write $M \in R^{p \times q}$ to indicate that $M$ is a $p \times q$ matrix with elements in $R . M^{\text {adj }}$ and det $M$ denote the classical adjoint and the determinant of $M \in R^{p \times p}$, respectively. Let $M^{*}$ denote the conjugate transpose of $M \in \mathbf{M}(\mathbb{C})$. For a matrix-valued function $G$, let us denote by $\left(s_{i},\left[\xi_{i}, \eta_{i}\right]\right)_{i=1}^{n}$ the tangential interpolation data of $\xi_{i}^{*} G\left(s_{i}\right)=\eta_{i}^{*}$ for $i=1, \ldots, n$.

The Euclidean norm of $v \in \mathbb{C}^{p}$ is defined by $\|v\|=\left(v^{*} v\right)^{1 / 2}$. For $M \in \mathbb{C}^{p \times q}$, its Euclidean induced norm is defined by $\|M\|=$ $\sup \left\{\|M v\|: v \in \mathbb{C}^{q}\right.$ with $\left.\|v\|=1\right\}$ and equals the largest singular value of $M$. For $G \in \mathbf{M}\left(\mathcal{H}^{\infty}\right)$, the $\mathcal{H}^{\infty}$ norm is defined as $\|G\|_{\infty}=$ $\sup _{s \in \mathbb{C}_{+}}\|G(s)\|$.

A matrix $U \in R^{p \times p}$ is unimodular if it has an inverse in $R^{p \times p} . D$ and $N$ in $\mathbf{M}\left(\mathcal{H}^{\infty}\right)$ are said to be left coprime if they satisfy the Bezout identity

$$
N X+D Y=I
$$

\section{PRoBlem StATEMENT}

Consider the closed-loop system shown in Fig. 1, where $P \in \mathbf{M}\left(\mathcal{F}^{\infty}\right)$ represents the plant and $C \in \mathbf{M}\left(\mathcal{F}^{\infty}\right)$ does the controller. The closed-loop system is defined to be internally stable if $\operatorname{det}(I+P C)=\operatorname{det}(I+C P) \neq 0$ and the transfer matrix $H(P, C)$ from $u_{1}, u_{2}$ to $e_{1}, e_{2}$ satisfies

$$
H(P, C)=\left[\begin{array}{cc}
(I+P C)^{-1} & -P(I+C P)^{-1} \\
C(I+P C)^{-1} & (I+C P)^{-1}
\end{array}\right] \in \mathbf{M}\left(\mathcal{H}^{\infty}\right) .
$$

We say that $C$ stabilizes $P$ if the closed-loop system is internally stable. Let $C(P)$ denote the set of all controllers stabilizing $P$. A plant $P$ is said to be stabilizable if $\varnothing(P) \neq \emptyset$ and $P$ is strongly stabilizable if $\mathbf{M}\left(\mathcal{H}^{\infty}\right) \cap \chi(P) \neq \emptyset$.

If $P$ and $C$ are real-rational and proper, then $P$ is strongly stabilizable if and only if $P$ satisfies the parity interlacing property [3], [15]. On the other hand, every stabilizable $P \in \mathbf{M}\left(\mathcal{F}^{\infty}\right)$ is known to be strongly stabilizable [16], but in this case, $C$ generally belongs not to $\mathbf{M}\left(\mathcal{R} \mathcal{H}^{\infty}\right)$ but to $\mathbf{M}\left(\mathcal{H}^{\infty}\right)$, which means $C(s) \in \mathbb{C}$ for real $s$.

Our problem is the following:

1) Problem II.1: Given a plant $P \in \mathbf{M}\left(\mathcal{F}^{\infty}\right)$, weighting matrices $W_{1}, W_{2} \in \mathbf{M}\left(\mathcal{H}^{\infty}\right)$, determine whether there exists a controller $C \in$ $\mathbf{M}\left(\mathcal{H}^{\infty}\right) \cap \mathscr{C}(P)$ such that

$$
\left\|W_{1}(I+P C)^{-1} W_{2}\right\|_{\infty}<1
$$

Also, if one exists, find such a controller.

The aim of this article is to obtain a sufficient condition and also a necessary condition for Problem II. 1 that can be checked by matrix computations. We also design stable controllers satisfying(II.2) under the sufficient condition.

In [13], the same problem has been studied. Here, we place more general constraints on the multivariable zeros of the plant than those in [13]. In the next section, we will discuss the difference of the constraints and address the nontrivial modifications arising from it.

\section{Sensitivity Reduction by Stable Controllers}

In this section, we prove that if the plant has unstable transmission zeros, strong stabilization is equivalent to tangential interpolation by a unimodular matrix in $\mathbf{M}\left(\mathcal{H}^{\infty}\right)$. In conjunction with the tangential Nevanlinna-Pick interpolation, this equivalence enables us to obtain both lower and upper bounds of the minimum sensitivity achievable by a stable controller.

\section{A. Strong Stabilization of MIMO Systems With Unstable Transmission Zeros}

Let us first study strong stabilization for MIMO systems only. By Lemma III.1 of [13], $C$ is stable and stabilizes $P$ with the left coprime factorization $P=D^{-1} N$ if and only if $C$ is a solution to the following problem:

1) Problem III.1: Suppose $D, N \in \mathbf{M}\left(\mathcal{H}^{\infty}\right)$ are left coprime. Find $C \in \mathbf{M}\left(\mathcal{H}^{\infty}\right)$ such that $(D+N C)^{-1} \in \mathbf{M}\left(\mathcal{H}^{\infty}\right)$.

Throughout this note, we assume that the following holds:

Assumption III.2: All entries of $N, D, X$, and $Y$ in (I.1) are meromorphic in $\mathbb{C}$. In addition, $N$ is square and $\operatorname{det} N$ has the form

$$
\operatorname{det} N=\phi N_{o} \text {, where } \phi \in \mathcal{R} \mathcal{H}^{\infty} \text { and } N_{o}, 1 / N_{o} \in \mathcal{H}^{\infty} \text {. }
$$

The rational function $\phi$ satisfies $\phi(\infty) \neq 0$ and has only simple zeros $z_{1}, \ldots, z_{n}$ in $\overline{\mathbb{C}}_{+}$. For $i=1, \ldots, n$, the left annihilating nonzero vector $v_{i} \in \mathbb{C}^{p}$ satisfying

$$
v_{i}^{*} N\left(z_{i}\right)=0
$$


is unique up to multiplication by a constant complex number.

In [13], it is assumed that the matrix-valued function $N$ can be factored as $N=\phi N_{o}$, where $\phi \in \mathcal{R} \mathcal{H}^{\infty}$ and $N_{o}, N_{o}^{-1} \in\left(\mathcal{H}^{\infty}\right)^{p \times p}$. Note that Assumption III.2 requires a factorization (III.1) of the scalarvalued function $\operatorname{det} N$.

We shall show that Problem III.1 is equivalent to the following problem:

2) Problem III.3: Suppose that $s_{1}, \ldots, s_{n} \in \overline{\mathbb{C}}_{+}$are distinct and that $\xi_{1}, \ldots, \xi_{n}, \eta_{1}, \ldots, \eta_{n} \in \mathbb{C}^{p}$. Find a unimodular matrix $U \in\left(\mathcal{H}^{\infty}\right)^{p \times p}$ such that all elements of $U$ are meromorphic in $\mathbb{C}$ and $\xi_{i}^{*} U\left(s_{i}\right)=\eta_{i}^{*}$ for $i=1, \ldots, n$.

Theorem III.4: Consider Problem III.1 under Assumption III.2. We restrict the solutions to matrices whose entries are meromorphic in $\mathbb{C}$. Then Problem III. 1 is equivalent to Problem III.3 with the interpolation data $\left(z_{i},\left[v_{i}, D\left(z_{i}\right)^{*} v_{i}\right]\right)_{i=1}^{n}$.

Furthermore, a solution $C$ to Problem III.1 and a solution $U$ to Problem III.3 satisfy the following equations:

$$
C=N^{-1}(U-D), \quad U=D+N C .
$$

Proof: Let $C \in\left(\mathcal{H}^{\infty}\right)^{p \times p}$ be a meromorphic solution to Problem III.1. Define $U$ by (III.3). Then $U$ and $U^{-1}$ belong to $\left(\mathcal{H}^{\infty}\right)^{p \times p}$ [13], Lemma III.1 and

$$
v_{i}^{*} U\left(z_{i}\right)=v_{i}^{*} D\left(z_{i}\right)+v_{i}^{*} N\left(z_{i}\right) C\left(z_{i}\right)=\left(D\left(z_{i}\right)^{*} v_{i}\right)^{*} .
$$

Thus $U$ is a solution to Problem III.3 with the interpolation data $\left(z_{i},\left[v_{i}, D\left(z_{i}\right)^{*} v_{i}\right]\right)_{i=1}^{n}$.

Conversely, let $U \in\left(\mathcal{H}^{\infty}\right)^{p \times p}$ be a solution to Problem III.3 with the interpolation data $\left(z_{i},\left[v_{i}, D\left(z_{i}\right)^{*} v_{i}\right]\right)_{i=1}^{n}$. Define $C$ by (III.3). Then $C$ satisfies $(D+N C)^{-1}=U^{-1} \in\left(\mathcal{H}^{\infty}\right)^{p \times p}$,

$$
N C=U-D \in\left(\mathcal{H}^{\infty}\right)^{p \times p}
$$

and

$$
v_{i}^{*}(N C)\left(z_{i}\right)=v_{i}^{*}\left(U\left(z_{i}\right)-D\left(z_{i}\right)\right)=0 .
$$

We prove $C \in\left(\mathcal{H}^{\infty}\right)^{p \times p}$ by (III.4) and (III.5) as follows. Define $\Upsilon=N C$. Then $\Upsilon \in\left(\mathcal{H}^{\infty}\right)^{p \times p}$ by (III.4) and $v_{i}^{*} \Upsilon\left(z_{i}\right)=0$ by (III.5). Since we have by Cramer's rule

$$
N^{\text {adj }} \cdot N=N \cdot N^{\text {adj }}=\operatorname{det} N \cdot I,
$$

it follows from the definition of $\Upsilon$ that

$$
\phi C=1 / N_{o} \cdot N^{\mathrm{adj}} \cdot \Upsilon \in\left(\mathcal{H}^{\infty}\right)^{p \times p}
$$

Also, we obtain the following equation:

$$
N^{\operatorname{adj}}\left(z_{i}\right) \Upsilon\left(z_{i}\right)=0, \quad i=1, \ldots, n
$$

This is because every row of $N^{\text {adj }}\left(z_{i}\right)$ is a constant multiple of $v_{i}^{*}$. To see this, let $N_{m}^{\text {adj }}\left(z_{i}\right)$ be the $m$-th row of $N^{\text {adj }}\left(z_{i}\right)$. Since

$$
N^{\operatorname{adj}}\left(z_{i}\right) N\left(z_{i}\right)=\left(\phi\left(z_{i}\right) N_{o}\left(z_{i}\right)\right) \cdot I=0
$$

by (III.6), we have $N_{m}^{\operatorname{adj}}\left(z_{i}\right) N\left(z_{i}\right)=0$ for $m=1, \ldots, p$. Thus, the uniqueness of $v_{i}$ in Assumption III.2 implies that $N_{m}^{\operatorname{adj}}\left(z_{i}\right)=k_{m} v_{i}^{*}$ for some $k_{m} \in \mathbb{C}$.

Since the unit $N_{o}$ have no unstable zero, (III.7) and(III.8) show

$$
(\phi C)\left(z_{i}\right)=0, \quad i=1, \ldots, n .
$$

Thus it suffices to prove $C \in\left(\mathcal{H}^{\infty}\right)^{p \times p}$ from the following three conditions: The unstable zeros $z_{1}, \ldots, z_{n}$ of $\phi$ are simple; $\phi C \in\left(\mathcal{H}^{\infty}\right)^{p \times p}$; and (III.9) holds.

Suppose $C \notin\left(\mathcal{H}^{\infty}\right)^{p \times p}$. Then, since $\phi C \in\left(\mathcal{H}^{\infty}\right)^{p \times p}$, the unstable poles of $C$ must be the zeros of $\phi$. Let $z_{i}$ be one of such poles. Since $\phi$ has only simple zeros in $\overline{\mathbb{C}}_{+}$, it follows that $(\phi C)\left(z_{i}\right) \neq 0$. This contradicts (III.9), and hence $C \in\left(\mathcal{H}^{\infty}\right)^{p \times p}$.

Prasanth [17] presents a method to find a unimodular matrix in $\left(\mathcal{R} \mathcal{H}^{\infty}\right)^{p \times p}$ satisfying tangential interpolation conditions. In [17], a result similar to Theorem III.4 is also developed for finite dimensional systems. The argument there makes use of the results of [10] and a state-space realization of the plant. Hence it is not applicable to the present situation. On the other hand, using Cramer's rule, we prove Theorem III.4 in a transfer-function approach.

Remark III.5: In [13], we have considered matrix-valued interpolation conditions $U\left(z_{i}\right)=D\left(z_{i}\right)$. This interpolation leads to the stringent assumption that all unstable zeros $z_{i}$ of the plant be blocking zeros, enabling us to handle such multivariable zeros in a way similar to that used for zeros of SISO systems. On the other hand, here we address tangential interpolation conditions $v_{i}^{*} U\left(z_{i}\right)=v_{i}^{*} D\left(z_{i}\right)$ so that the plant is allowed to have unstable transmission zeros.

\section{B. Strong Stabilization With Sensitivity Reduction}

Let us next proceed to the problem of strong stabilization with sensitivity reduction. We further place the next assumptions on $W_{1}, W_{2}$, and $D$ :

Assumption III.6: All elements of All $W_{1}$ and $W_{2}$ are meromorphic functions in $\mathbb{C}$. Both $W_{1}$ and $W_{1}^{-1}$ belong to $\mathbf{M}\left(\mathcal{H}^{\infty}\right)$. Let $D W_{2}$ have a factorization $D W_{2}=\left(D W_{2}\right)_{c o} \cdot\left(D W_{2}\right)_{c i}$, where $\left(D W_{2}\right)_{c o}$ is co-outer and $\left(D W_{2}\right)_{c i}$ is co-inner. $\left(D W_{2}\right)_{c o}$ and $\left(D W_{2}\right)_{c o}^{-1}$ also belong to $\mathbf{M}\left(\mathcal{H}^{\infty}\right)$.

By extending the results of the previous subsection, we shall prove that Problem II.1 is equivalent to the following Problem III.7 under Assumptions III.2 and III.6. The only difference between Problems III.3 and III.7 is that the latter problem requires that the $\mathcal{H}^{\infty}$ norm of a solution be less than one.

1) Problem III.7: Suppose $s_{1}, \ldots, s_{n} \in \overline{\mathbb{C}}_{+}$are distinct. Let $\xi_{1}, \ldots, \xi_{n}, \eta_{1}, \ldots, \eta_{n} \in \mathbb{C}^{p}$. Find a unimodular matrix $F \in\left(\mathcal{H}^{\infty}\right)^{p \times p}$ such that all elements of $F$ are meromorphic in $\mathbb{C},\|F\|_{\infty}<1$, and

$$
\xi_{i}^{*} F\left(s_{i}\right)=\eta_{i}^{*}, \quad i=1, \ldots, n .
$$

Theorem III.8: Consider Problem II.1. Suppose there exist $D, N \in$ $\mathbf{M}\left(\mathcal{H}^{\infty}\right)$ such that $P=D^{-1} N$, and let Assumptions III.2 and III.6 hold. Define the vector pairs $\left(\xi_{i}, \eta_{i}\right)$ by

$$
\xi_{i}=\left(D\left(z_{i}\right) W_{1}^{-1}\left(z_{i}\right)\right)^{*} v_{i}, \quad \eta_{i}=\left(\left(D W_{2}\right)_{c o}\left(z_{i}\right)\right)^{*} v_{i}
$$

for $i=1, \ldots, n$. If there exists a solution $F$ to Problem III.7 with the interpolation data $\left(z_{i},\left[\xi_{i}, \eta_{i}\right]\right)_{i=1}^{n}$, then a solution to Problem II.1 is given by

$$
C=N^{-1}\left(D W_{2}\right)_{{ }_{c o}} F^{-1} W_{1}-P^{-1}
$$

conversely, if there exists a solution $C$ to Problem II.1 and if all entries of $C$ are meromorphic in $\mathbb{C}$, then

$$
F=W_{1}(D+N C)^{-1}\left(D W_{2}\right)_{c o}
$$

is a solution to Problem III.7 with the interpolation data $\left(z_{i},\left[\xi_{i}, \eta_{i}\right]\right)_{i=1}^{n}$. 
Proof: Let a unimodular matrix $F \in\left(\mathcal{H}^{\infty}\right)^{p \times p}$ be a solution to Problem III.7 with the interpolation data $\left(z_{i},\left[\xi_{i}, \eta_{i}\right]\right)_{i=1}^{n}$. Define $C$ by (III.12).

To prove $C \in\left(\mathcal{H}^{\infty}\right)^{p \times p} \cap \mathscr{C}(P)$, it suffices to show, by Theorem III.4, that $U$ defined by

$$
U=D+N C=\left(D W_{2}\right)_{c o} F^{-1} W_{1}
$$

satisfies $U, U^{-1} \in\left(\mathcal{H}^{\infty}\right)^{p \times p}$ and

$$
v_{i}^{*} U\left(z_{i}\right)=v_{i}^{*} D\left(z_{i}\right)
$$

for $i=1, \ldots, n$.

Since $\left(D W_{2}\right)_{c o}, F$, and $W_{1}$ are unimodular, it follows from (III.14) that $U, U^{-1} \in\left(\mathcal{H}^{\infty}\right)^{p \times p}$. Additionally, since $\xi_{i}^{*}=\eta_{i}^{*} F\left(z_{i}\right)^{-1}$, we have

$$
v_{i}^{*} U\left(z_{i}\right)=\eta_{i}^{*} F^{-1}\left(z_{i}\right) W_{1}\left(z_{i}\right)=\xi_{i}^{*} W_{1}\left(z_{i}\right)=v_{i}^{*} D\left(z_{i}\right) .
$$

Hence we obtain $C \in\left(\mathcal{H}^{\infty}\right)^{p \times p} \cap \mathscr{C}(P)$.

Moreover, simple calculations show

$$
\begin{aligned}
& \left\|W_{1}(1+P C)^{-1} W_{2}\right\|_{\infty} \\
& \quad=\left\|W_{1}(D+N C)^{-1}\left(D W_{2}\right)_{c o} \cdot\left(D W_{2}\right)_{c i}\right\|_{\infty} \\
& \quad=\|F\|_{\infty} .
\end{aligned}
$$

Thus $C$ is a solution to Problem II.1.

Conversely, suppose $C$ is a solution to Problem II.1 and all the entries are meromorphic. Define $F$ by (III.13). Then, since $U$ in (III.14) satisfies $U, U^{-1} \in\left(\mathcal{H}^{\infty}\right)^{p \times p}$ by Theorem III.4, it follows that $F$, $F^{-1} \in\left(\mathcal{H}^{\infty}\right)^{p \times p}$. $F$ also satisfies $\|F\|_{\infty}<1$ by (III.15). In addition, since

$$
\begin{aligned}
\xi_{i}^{*} F\left(z_{i}\right) & =v_{i}^{*} D\left(z_{i}\right)(D+N C)^{-1}\left(z_{i}\right)\left(D W_{2}\right)_{c o}\left(z_{i}\right) \\
& =v_{i}^{*}\left(D W_{2}\right)_{c o}\left(z_{i}\right) \\
& =\eta_{i}^{*}
\end{aligned}
$$

by (III.11),(III.14), and(III.2), we obtain(III.10). Thus $F$ is a solution to Problem III.7 with the interpolation data $\left(z_{i},\left[\xi_{i}, \eta_{i}\right]\right)_{i=1}^{n}$.

Theorem III.8 suggests that the problem of strong stabilization with sensitivity reduction is equivalent to Problem III.7. The natural question then arises: Is this interpolation problem solvable? Since the solution to Problem III.7 must be unimodular, it is difficult to give a necessary and sufficient condition. Here we derive a sufficient condition and a necessary condition for Problem III.7 via the tangential Nevanlinna-Pick interpolation.

To proceed, we recall the tangential Nevanlinna-Pick interpolation with boundary conditions:

2) Problem III.9 [10]: Suppose $\alpha_{1}, \ldots \alpha_{n} \in \mathbb{C}_{+}$and $j \omega_{1}, \ldots, j \omega_{m} \in j B B R$ are distinct. Let vector pairs $\left(\xi_{i}, \eta_{i}\right)$ and $\left(x_{k}, y_{k}\right)$ in $\mathbb{C}^{p} \times \mathbb{C}^{q}$ satisfy

$$
\begin{aligned}
\left\|\xi_{i}\right\|-\left\|\eta_{i}\right\|>0, & i=1, \ldots, n, \\
\left\|x_{k}\right\|-\left\|y_{k}\right\|>0, & k=1, \ldots, m .
\end{aligned}
$$

Find $\Phi \in\left(\mathcal{H}^{\infty}\right)^{p \times q}$ such that $\|\Phi\|_{\infty}<1$ and

$$
\begin{aligned}
\xi_{i}^{*} \Phi\left(\alpha_{i}\right) & =\eta_{i}^{*}, & i=1, \ldots, n, \\
x_{k}^{*} \Phi\left(j \omega_{k}\right) & =y_{k}^{*}, \quad & k=1, \ldots, m .
\end{aligned}
$$

Problem III.9 is solvable if and only if the Pick matrix consisting of the interpolation data $\left(\alpha_{i},\left[\xi_{i}, \eta_{i}\right]\right)_{i=1}^{n}$ at the interior points of $\mathbb{C}_{+}$ is positive definite [10]. Calculation methods of the interpolant $\Phi$ are developed in [10], [18].
First we derive a necessary condition. Define

$$
\begin{array}{r}
\rho_{\text {inf }}=\inf \left\{\left\|W_{1}(I+P C)^{-1} W_{2}\right\|_{\infty}: C \in \mathbf{M}\left(\mathcal{H}^{\infty}\right) \cap \varnothing(P),\right. \\
\text { all entries of } C \text { are meromorphic }\} .
\end{array}
$$

From Theorem III.8, we deduce the next result providing a lower bound of $\rho_{\text {inf }}$.

Corollary III.10: Consider Problem II.1 under the same hypotheses of Theorem III.8. For a given $\rho>0$, if there does not exist $G \in\left(\mathcal{H}^{\infty}\right)^{p \times p}$ such that $\|G\|_{\infty}<1$ and $\xi_{i}^{*} G\left(z_{i}\right)=\eta_{i}^{*} / \rho$ for $i=1, \ldots, n$, then $\rho_{\text {inf }}$ in (III.16) satisfies $\rho_{\text {inf }} \geq \rho$.

Let us next develop a sufficient condition and a design method of stable stabilizing controllers that achieve low sensitivity. We extend the technique of [13], [19] to the tangential interpolation case.

Lemma III.11: Consider Problem III.7. Let $\lambda \in \mathbb{C}$ and define

$$
\zeta_{i}=\frac{2}{\bar{\lambda}} \eta_{i}-\xi_{i}, \quad i=1, \ldots, n .
$$

If $G \in\left(\mathcal{H}^{\infty}\right)^{p \times p}$ satisfies $\|G\|_{\infty}<1$ and

$$
\xi_{i}^{*} G\left(z_{i}\right)=\zeta_{i}^{*}, \quad i=1, \ldots, n
$$

then $F$ defined by

$$
F=\frac{\lambda}{2}(G+I)
$$

is unimodular and satisfies $\|F\|_{\infty}<|\lambda|$ and the interpolation constraints (III.10).

Proof: Since $\|G\|_{\infty}<1$, it follows that $G+I$ is unimodular by the small gain theorem, and that $\|G+I\|_{\infty}<2$ by the triangle inequality $\|G+I\|_{\infty} \leq\|G\|_{\infty}+\|I\|_{\infty}$. Therefore $F$ and $F^{-1}$ belong to $\left(\mathcal{H}^{\infty}\right)^{p \times p}$, and $\|F\|_{\infty}<|\lambda|$. By (III.17),(III.18), and(III.19), $F$ also satisfies (III.10).

Combining Theorem III.8 with Lemma III.11, we obtain an upper bound of $\rho_{\text {inf }}$ and a stable controller achieving it.

Theorem III.12: Consider Problem II.1 under the same assumptions and definitions as in Theorem III.8 and Lemma III.11. If there exists $G \in\left(\mathcal{H}^{\infty}\right)^{p \times p}$ such that $\|G\|_{\infty}<1$ and (III.18) holds, then $\rho_{\text {inf }}$ in (III.16) satisfies $\rho_{\text {inf }}<|\lambda|$ and a solution to Problem II.1 is given by

$$
C=\frac{2}{\lambda} N^{-1}\left(D W_{2}\right)_{c o}(G+I)^{-1} W_{1}-P^{-1} .
$$

Note that Theorem III.12 and Corollary III.10 give upper and lower bounds of the minimum sensitivity $\rho_{\text {inf }}$ by iterative computations of the associated Pick matrices.

We conclude this section with two remarks on Assumption III.2.

Remark III.13:

1) In this section, we have assumed that all functions are meromorphic in $\mathbb{C}$ because $\mathcal{H}^{\infty}$ functions do not necessarily have a finite value on the imaginary axis. If the unstable zeros of $\operatorname{det} N$ are not on the imaginary axis, then we remove the assumption that all elements of the transfer matrices are meromorphic.

2) We have assumed that det $N$ has only simple zeros in $\overline{\mathbb{C}}_{+}$, but the results in this section can be generalized to the case in which $\operatorname{det} N$ has unstable zeros of higher order. In this case, we need to introduce interpolation conditions involving derivatives of $N$ and $D$.

For example, let $z_{i}$ be a unstable zero of order $2 \operatorname{of} \operatorname{det} N$, and suppose that $v_{i}$ and $\bar{v}_{i}$ are unique vectors such that $v_{i}^{*} N\left(z_{i}\right)=0$ and $v_{i}^{*} N^{\prime}\left(z_{i}\right)+\bar{v}_{i}^{*} N\left(z_{i}\right)=0$. Then the interpolation conditions of $U$ in Theorem III. 4 are given by

$$
\begin{aligned}
v_{i}^{*} U\left(z_{i}\right) & =v_{i}^{*} D\left(z_{i}\right)=: w_{i}^{*}, \\
v_{i}^{*} U^{\prime}\left(z_{i}\right)+\bar{v}_{i}^{*} U\left(z_{i}\right) & =\bar{v}_{i}^{*} D\left(z_{i}\right)+v_{i}^{*} D^{\prime}\left(z_{i}\right)=: \bar{w}_{i}^{*} .
\end{aligned}
$$


Also, if we take $\left(D W_{2}\right)_{c o}=I$ for simplicity, $F$ in Theorem III.8 must satisfy

$$
\begin{gathered}
\xi_{i}^{*} F\left(z_{i}\right)=v_{i}^{*}, \\
\bar{\xi}_{i}^{*} F\left(z_{i}\right)+\xi_{i}^{*} F^{\prime}\left(z_{i}\right)=\bar{v}_{i}^{*}
\end{gathered}
$$

where

$$
\begin{aligned}
& \xi_{i}=W_{1}^{-1}\left(z_{i}\right)^{*} w_{i}, \\
& \bar{\xi}_{i}=W_{1}^{-1}\left(z_{i}\right)^{*} \bar{w}_{i}+\left(W_{1}^{-1}\right)^{\prime}\left(z_{i}\right)^{*} w_{i} .
\end{aligned}
$$

Since these interpolation conditions are immediate consequences of

$$
N^{\text {adj }} N^{\prime}+\left(N^{\text {adj }}\right)^{\prime} N=(\operatorname{det} N)^{\prime}
$$

and

$$
\left(U^{-1}\right)^{\prime}=-U^{-1} U^{\prime} U^{-1}
$$

we omit the details of the proof.

\section{NumERICAL EXAMPLES}

In this section, we present a numerical example to show the effectiveness of the results. We also apply the proposed method to a repetitive control system [20], [21] with a coprime factorization technique on MIMO systems whose infinite dimensional part is scalar. See [22] for the applications of repetitive control systems.

1) Example IV.1: We consider sensitivity reduction by a stable stabilizing controller for the following infinite dimensional system and weighting functions:

$$
\begin{aligned}
& P(s)=\left[\begin{array}{cc}
\frac{\left(s-z_{1}\right)\left(s-z_{2}\right)}{(s+1)^{2}\left(1+3 e^{-2 s}\right)} & \frac{e^{-s}}{s+2} \\
0 & \frac{(s+1)^{2}}{(s-1)\left(s-e^{-s}+2\right)}
\end{array}\right] \\
& W_{1}(s)=\frac{s+1}{10 s+1} I, \quad W_{2}(s)=I
\end{aligned}
$$

where $z_{1}, z_{2} \in \mathbb{C}_{+}$are distinct.

Let us begin by finding left coprime $D, N \in\left(\mathcal{H}^{\infty}\right)^{2 \times 2}$ such that $D^{-1} N=P$. First, applying the factorization method of [23] to each element of $P$, we show that $P$ can be factored as $P=D^{-1} N$, where

$$
\begin{gathered}
D(s)=\left[\begin{array}{cc}
\frac{1+3 e^{-2 s}}{e^{-2 s}+3} & 0 \\
0 & \frac{s-1}{s+1}
\end{array}\right], \\
N(s)=\left[\begin{array}{cc}
\frac{\left(s-z_{1}\right)\left(s-z_{2}\right)}{(s+1)^{2}\left(e^{-2 s}+3\right)} & \frac{1+3 e^{-2 s}}{e^{-2 s}+3} \cdot \frac{e^{-s}}{s+2} \\
0 & \frac{s+1}{s-e^{-s}+2}
\end{array}\right] .
\end{gathered}
$$

The unstable zeros of $\operatorname{det} N$ are $z_{1}$ and $z_{2}$. The vectors $v_{i}$ given by

$$
v_{i}=\left[\begin{array}{ll}
-\frac{e^{-2 z_{i}}+3}{1+3 e^{-2 z_{i}}} \cdot \frac{z_{i}+2}{e^{-z_{i}}} & \frac{z_{i}-e^{-z_{i}}+2}{z_{i}+1}
\end{array}\right]^{*}, i=1,2
$$

satisfy $v_{i}^{*} N\left(z_{i}\right)=0$ and they are unique up to multiplication by a constant complex number.

Next, from the same argument leading to $C \in\left(\mathcal{H}^{\infty}\right)^{p \times p}$ in Theorem III.4, we see that $D$ and $N$ are left coprime if and only if there exists $Y \in\left(\mathcal{H}^{\infty}\right)^{2 \times 2}$ satisfying the interpolation conditions $v_{i}^{*} D\left(z_{i}\right) Y\left(z_{i}\right)=v_{i}^{*}$ for $i=1,2$. This problem is called the tangential Lagrange interpolation [10], Chapter 16. We can check the existence of such $Y$ by the tangential Nevanlinna-Pick interpolation with the scaling of the interpolation data.

We take $0<z_{1} \leq 3$ and $z_{2}=5$. Fig. 2 shows the relationship between the unstable transmission zero $z_{1}$ and the minimum sensitivity $\rho_{\text {inf }}$ in (III.16). In Fig. 2, the solid line indicates an upper bound of $\rho_{\text {inf }}$ derived by Theorem III.12. The dashed line shows a lower bound of $\rho_{\text {inf }}$ obtained by Corollary III.10. From Fig. 2, we see that an unstable

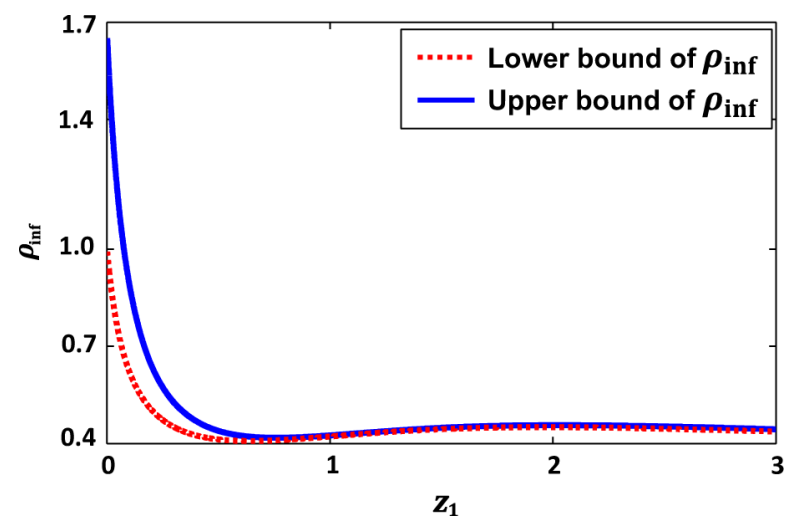

Fig. 2. Unstable zero $z_{1}$ versus minimum sensitivity $\rho_{\text {inf }}$.

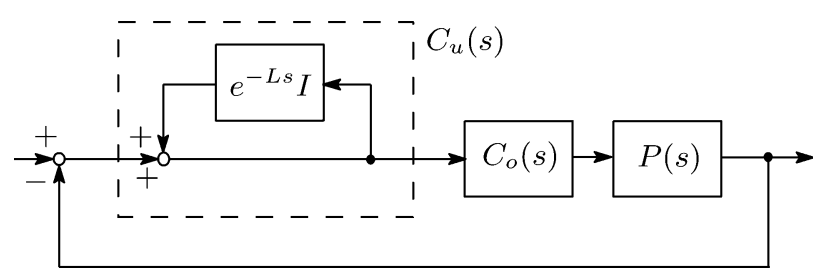

Fig. 3. Repetitive control system.

pole-zero cancellation at $s=1$ in $\operatorname{det} P$ does not affect strong stabilization with sensitivity reduction. This is because $z_{1}$ is not a blocking zero but a simple transmission zero and it is not in the same input nor output channel as the pole at $s=1$.

2) Example IV.2: (Application to repetitive control systems)

Consider the repetitive control system in Fig. 3, where $P$ is a finite dimensional plant and $C_{u}=1 /\left(1-e^{-L s}\right) \cdot I$ is the internal model of any periodic signals with period $L$.

Note that if we use the internal model of the type $1 /\left(1-e^{-L s}\right)$, then the closed-loop internal stability cannot be achieved for strictly proper plant [21, Theorem 5.12]. Also, such an internal model leads to the potential loss of the w-stability [24, Section 8] of the closed-loop system. So it is practical to construct modified repetitive controllers [20]-[22]. To illustrate our results, however, we do not proceed along this line.

For a given $P$ and $C_{u}$, we design $C_{o}$ to meet performance requirements. Here let us find $C_{o} \in \mathbf{M}\left(\mathcal{H}^{\infty}\right)$ yielding exponential stability and low sensitivity of the closed loop system. By the same argument as in [13], in order to do this, we study Problem II.1 with $\tilde{P}(s)=$ $C_{u}(s-\varepsilon) P(s-\varepsilon)$ for some $\varepsilon>0$. If we find the solution $\tilde{C}$ to the problem, then we design $C_{o}$ by $C_{o}(s)=\tilde{C}(s+\varepsilon)$. Since $\tilde{C} \in$ $\mathbf{M}\left(\mathcal{H}^{\infty}\right)$, it follows that $C_{o}$ is analytic and bounded in the region $\{s \in \mathbb{C} \mid \operatorname{Re} s>-\varepsilon\}$.

When we apply the proposed method to infinite dimensional systems, we first raise the following question: How do we obtain a left coprime factorization of general MIMO infinite dimensional systems? If the infinite dimensional part of the systems is scalar, we can answer this question affirmatively by using a factorization of the finite dimensional part.

Theorem IV.3: Let Assumption III.2 hold for $D, N \in\left(\mathcal{R H}^{\infty}\right)^{p \times p}$. Suppose $f \in \mathcal{H}^{\infty}$ is meromorphic in $\mathbb{C}$ and satisfies $f\left(z_{i}\right) \neq 0$ for every $i$. Then $f D$ and $N$ are left coprime.

Proof: By (I.1) and (III.6), we have

$$
N^{\text {adj }} \cdot(I-D Y)=\operatorname{det} N \cdot X \text {. }
$$


Additionally, for every $m$, the $m$-th row of $N^{\text {adj }}\left(z_{i}\right)$ is $k_{m} v_{i}^{*}$ with some $k_{m} \in \mathbb{C}$ by the proof of Theorem III.4. Hence

$$
v_{i}^{*}\left(I-D\left(z_{i}\right) Y\left(z_{i}\right)\right)=0
$$

or $k_{1}, \ldots, k_{p}=0$, i.e., $N^{\text {adj }}\left(z_{i}\right)=0$.

Let us first prove (IV.1) holds. Suppose $N^{\text {adj }}\left(z_{i}\right)=0$. Then there exists $\overline{N^{\text {adj }}} \in\left(\mathcal{R} \mathcal{H}^{\infty}\right)^{p \times p}$ such that $N^{\text {adj }}(s)=\left(s-z_{i}\right) \overline{N^{\operatorname{adj}}}(s)$. By Assumption III.2, there is also $\overline{\operatorname{det} N} \in \mathcal{R} \mathcal{H}^{\infty}$ such that $\operatorname{det} N(s)=$ $\left(s-z_{i}\right) \overline{\operatorname{det} N}(s)$ and $\overline{\operatorname{det} N}\left(z_{i}\right) \neq 0$. Therefore, since

$$
N \cdot \overline{N^{\operatorname{adj}}}=\overline{\operatorname{det} N} \cdot I
$$

by (III.6), we have

$$
\overline{\operatorname{det} N}\left(z_{i}\right) \cdot v_{i}^{*}=v_{i}^{*} N\left(z_{i}\right) \overline{N^{\operatorname{adj}}}\left(z_{i}\right)=0 .
$$

This contradicts $\overline{\operatorname{det} N}\left(z_{i}\right) \neq 0$ and $v_{i} \neq 0$; hence (IV.1) always holds.

Next we observe a sufficient condition for the left coprimeness of $f D$ and $N$. Let $Y_{o} \in\left(\mathcal{H}^{\infty}\right)^{p \times p}$ satisfy

$$
v_{i}^{*}\left(I-f\left(z_{i}\right) D\left(z_{i}\right) Y_{o}\left(z_{i}\right)\right)=0, \quad i=1, \ldots, n .
$$

Define

$$
X_{o}=N^{-1}\left(I-f D Y_{o}\right)
$$

Then $X_{o}$ satisfies the Bezout identity

$$
N X_{o}+f D Y_{o}=I .
$$

In addition, we have $X_{o} \in\left(\mathcal{H}^{\infty}\right)^{p \times p}$. This proof follows the same line as that of $C \in\left(\mathcal{H}^{\infty}\right)^{p \times p}$ in Theorem III.4, so it is omitted. Thus if there is $Y_{o}$ satisfying (IV.2), $f D$ and $N$ are left coprime.

The argument given above suggests that, to show the left coprimeness of $f D$ and $N$, it suffices to prove the following: If there exists $Y \in\left(\mathcal{R} \mathcal{H}^{\infty}\right)^{p \times p}$ such that $\xi_{i}^{*} Y\left(z_{i}\right)=\eta_{i}^{*}$ for $i=1, \ldots, n$, then there also exists $Y_{o} \in\left(\mathcal{H}^{\infty}\right)^{p \times p}$ such that

$$
\xi_{i}^{*}\left(a_{i} Y_{o}\left(z_{i}\right)\right)=\eta_{i}^{*}, \quad i=1, \ldots, n,
$$

where $\xi_{i}=D^{*}\left(z_{i}\right) v_{i}, \eta_{i}=v_{i}$, and $a_{i}=f\left(z_{i}\right)$.

Since $a_{i}=f\left(z_{i}\right) \neq 0$, we construct by the Lagrange interpolation [25] a rational function $g \in \mathcal{H}^{\infty}$ such that $g\left(z_{i}\right)=1 / a_{i}$ for $i=$ $1, \ldots, n$. Now define $Y_{o}=g Y \in\left(\mathcal{H}^{\infty}\right)^{p \times p}$. Then, since

$$
Y_{o}\left(z_{i}\right)=\frac{1}{a_{i}} Y\left(z_{i}\right), \quad i=1, \ldots, n,
$$

it follows that $Y_{o}$ satisfies (IV.3). Therefore, $f D$ and $N$ are left coprime.

Theorem IV. 3 asserts that if there is no unstable hidden modes in the product $\tilde{P}(s)=C_{u}(s-\varepsilon) P(s-\varepsilon)$, then $\tilde{P}$ has the following left coprime factorization:

$$
\tilde{P}(s)=\left(C_{u}(s-\varepsilon) D(s)\right)^{-1} \cdot N(s),
$$

where $D, N \in\left(\mathcal{R H}^{\infty}\right)^{p \times p}$ are left coprime and satisfy $P(s-\varepsilon)=$ $D^{-1}(s) N(s)$.

Finally, taking $\varepsilon=0.1, L=1$, and

$$
P(s)=\left[\begin{array}{cc}
\frac{s-1}{s-2} & \frac{1}{s-5} \\
\frac{1}{s+8} & \frac{s}{s-5}
\end{array}\right], \quad W_{1}(s)=\frac{s+1}{10 s+1} I, \quad W_{2}=I
$$

we study Problem II.1 for $\tilde{P}$ in (IV.4), $W_{1}$, and $W_{2}$. Note that this example is different from that in [13], where all unstable zeros of $P$ must be blocking zeros. The plant $P$ in (IV.5) has two unstable transmission zeros: 0.846 and 0.291 .

By Theorem III.12 and Corollary III.10, we compute both upper and lower bounds of $\rho_{\text {inf }}$ in (III.16) with $\tilde{P} ; 0.6998 \leq \rho_{\text {inf }} \leq 0.7176$.
A solution $\tilde{C} \in\left(\mathcal{H}^{\infty}\right)^{2 \times 2}$ achieving the upper bound $\rho=0.7176$ is given by $\tilde{C}=N^{-1} D_{c o} F^{-1} W_{1}-\tilde{P}$, where $D_{c o}$ is a co-outer matrix of $D$ and $F$ is a solution to Problem III.7. $D_{c o}$ and $F$ are given by

$$
\begin{gathered}
D_{c o}(s) \approx\left[\begin{array}{c}
\frac{0.7071(s+7.862)(s+3.814)(s+1.992)}{(s+3.612)(s+1.698)(s+7.956)} \\
\frac{-0.0709(s-33.59)(s+1.58)}{(s+3.612)(s+1.698)(s+7.956)} \\
\frac{-0.0709(s+9.094)(s+7.398)}{(s+3.612)(s+1.698)(s+7.956)} \\
\frac{0.7071(s+7.995)(s+4.984)(s+1.72)}{(s+3.612)(s+1.698)(s+7.956)}
\end{array}\right] . \\
F(s) \approx\left[\begin{array}{cc}
\frac{0.6180(s+0.06924)}{s+0.9034} & \frac{0.2463(s+0.0555)}{s+0.9034} \\
\frac{-0.09049(s-0.9017)}{s+0.9034} & \frac{0.4234(s+1.536)}{s+0.9034}
\end{array}\right] .
\end{gathered}
$$

\section{CONCLUding REMARKS}

We have studied the problem of strong stabilization with sensitivity reduction for MIMO infinite dimensional systems. The systems are allowed to have infinitely many poles in $\mathbb{C}_{+}$but finitely many transmission zeros in $\overline{\mathbb{C}}_{+}$. Since we have derived only a sufficient condition and a necessary condition, the problem has not yet been fully solved. However the proposed method gives both upper and lower bounds of the minimum sensitivity via the tangential Nevanlinna-Pick interpolation. Hence we can obtain these bounds by iterative computations of the associated Pick matrices. We have also designed stable controllers attaining such an upper bound. In addition, we have presented two numerical examples. The second example illustrates practical application to a repetitive control system. Sensitivity minimization may not always lead to robust controllers, so future works include bounding other closed-loop transfer functions in (II.1) as well, e.g., mixed sensitivity reduction.

\section{REFERENCES}

[1] J. C. Doyle, B. A. Francis, and A. Tannenbaum, Feedback Control Theory. New York: Macmillan, 1992.

[2] D. C. MacMartin and J. P. How, "Implementation and prevention of unstable optimal compensators," in Proc. ACC, 1994.

[3] M. Vidyasagar, Control System Synthesis: A Factorization Approach. Cambridge, MA: MIT Press, 1985.

[4] R. H. Middleton, "Trade-offs in linear control system design," Automatica, vol. 27, pp. 281-292, 1991.

[5] A. N. Moser, "Designing controllers for flexible structures with $\mathrm{H}$-infinity/ $\mu$-synthesis," IEEE Control Syst., vol. 13, pp. 79-89, 1993.

[6] A. Cavallo, G. D. Maria, C. Natale, and S. Pirozzi, "Robust control of flexible structures with stable bandpass controllers," Automatica, vol. 44, pp. 1251-1260, 2008

[7] A. Sarjas, R. Svecko, and A. Chowdhury, "Strong stabilization servo controller with optimization of performance criteria," ISA Trans., vol. 50, pp. 419-431, 2011.

[8] H.U. Ünal and A. Iftar, "Stable $\mathcal{H}^{\infty}$ flow controller design using approximation of FIR filters," IEEE Trans. Instrum. Meas. Control, vol. 34, pp. 3-25, 2012

[9] C. Foias, H. Özbay, and A. Tannenbaum, Robust Control of Infinite Dimensional Systems: Frequency Domain Methods. London, U.K.: Springer-Verlag, 1996.

[10] J. A. Ball, I. Gohberg, and L. Rodman, Interpolation of Rational Matrix Functions. Basel: Birkhäuser-Verlag, 1990.

[11] S. Gümüssoy and H. Özbay, "Sensitivity minimization by strongly stabilizing controllers for a class of unstable time-delay systems," IEEE Trans. Automat. Control, vol. 54, pp. 590-595, 2009.

[12] M. Wakaiki, Y. Yamamoto, and H. Özbay, "Stable controllers for robust stabilization of systems with infinitely many unstable poles," Syst. Control Lett., vol. 62, pp. 511-516, 2013.

[13] M. Wakaiki, Y. Yamamoto, and H. Özbay, "Sensitivity reduction by strongly stabilizing controllers for MIMO distributed parameter systems," IEEE Trans. Autom. Control, vol. 57, pp. 2089-2094, 2012.

[14] P. Delsarte, Y. Genin, and Y. Kamp, "The Nevanlinna-Pick problem for matrix-valued functions," SIAM J. Appl. Math., vol. 36, pp. 47-61, 1979.

[15] D. Youla, J. Bongiorno, and C. Lu, "Single-loop feedback stabilization of linear multivariable dynamical plants," Automatica, vol. 10, pp. 159-173, 1974. 
[16] A. Quadrat, "On a general structure of the stabilizing controllers based on stable range," SIAM J. Control Optim., vol. 42, pp. 2264-2285, 2004.

[17] R. K. Prasanth, "Two-sided tangential interpolation with real rational units in "jo," Automatica, vol. 34, pp. 861-874, 1998.

[18] H. Dym, "A basic interpolation problem," in Holomorphic Space. : Cambridge University Press, 1998, pp. 381-423.

[19] H. Ito, H. Ohmori, and A. Sano, "Design of stable controllers attaining low $H^{\infty}$ weighted sensitivity," IEEE Trans. Autom. Control, vol. 38, pp. 485-488, 1993.

[20] S. Hara, Y. Yamamoto, T. Omata, and M. Nakano, "Repetitive control system: A new type servo system for periodic exogenous signals," IEEE Trans. Autom. Control, vol. 33, pp. 659-668, 1988.

[21] Y. Yamamoto and S. Hara, "Relationships between internal and external stability for infinite-dimensional systems with applications to a servo problem," IEEE Trans. Autom. Control, vol. 33, pp. 1044-1052, 1988.

[22] L. Cuiyan, Z. Dongchun, and Z. Xianyi, "A survey of repetitive control," in Proc. IEEE/RSJ IROS 2004, 2004

[23] S. Gümüssoy, "Coprime-inner/outer factorization of SISO time-delay systems and FIR structure of their optimal $H$-infinity controllers," Int. J. Robust Nonlinear Control, vol. 22, pp. 981-998, 2012.

[24] T. T. Georgiou and M. C. Smith, "Graphs, causality and sstabilizability: linear, shift-invariant systems on $L_{2}[0, \infty)$," Math. Control Signals Syst., vol. 6, pp. 195-223, 1993.

[25] P. Dorato, Analytic Feedback Design: An Interpolation Approach. Pacific Grove, CA: Brooks/Col, 2000.

\section{A Limiting Property of the Matrix Exponential}

Sebastian Trimpe, Member, IEEE, and

Raffaello D'Andrea, Fellow, IEEE

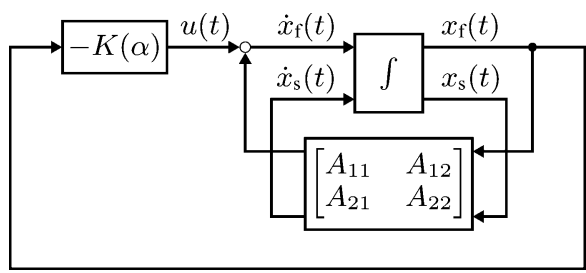

Fig. 1. Linear system with feedback on the first part of the state vector, the "fast" states $x_{\mathrm{f}}$.

in the limit as $K(\alpha)$ grows large for $\alpha \rightarrow \infty$ in some sense to be made precise later. All matrices are complex, and $\alpha$ is a real parameter. For different classes of $K(\alpha)$, we derive sufficient (and in one case also necessary) conditions on $K(\alpha)$ such that, for all $t>0$

$$
\lim _{\alpha \rightarrow \infty} \exp \left(\left[\begin{array}{cc}
A_{11}-K(\alpha) & A_{12} \\
A_{21} & A_{22}
\end{array}\right] t\right)=\left[\begin{array}{cc}
0 & 0 \\
0 & e^{A_{22} t}
\end{array}\right] .
$$

That is, we are interested in conditions guaranteeing that the coupling blocks $(1,2)$ and $(2,1)$ vanish (in addition to the $(1,1)$-block).

In addition to being an interesting matrix problem, the result can be applied to control systems that exhibit significantly different time scales, such as systems with high-gain feedback on some states. For example, consider the system

$$
\begin{aligned}
& \dot{x}_{\mathrm{f}}(t)=A_{11} x_{\mathrm{f}}(t)+A_{12} x_{\mathrm{s}}(t)+u(t) \\
& \dot{x}_{\mathrm{s}}(t)=A_{21} x_{\mathrm{f}}(t)+A_{22} x_{\mathrm{s}}(t)
\end{aligned}
$$

with static feedback on the states $x_{\mathrm{f}}(t)$ (index $\mathrm{f}$ for "fast" and $\mathrm{s}$ for "slow"),

$$
u(t)=-K(\alpha) x_{\mathrm{f}}(t) .
$$

Abstract-A limiting property of the matrix exponential is proven: if the
$(1,1)$-block of a 2-by-2 block matrix becomes "arbitrarily small" in a limiting process, the matrix exponential of that matrix tends to zero in the $(1,1)-,(1,2)-$, and $(2,1)$-blocks. The limiting process is such that either the log norm of the $(1,1)$-block goes to negative infinity, or, for a certain polynomial dependency, the matrix associated with the largest power of the variable that tends to infinity is stable. The limiting property is useful for simplification of dynamic systems that exhibit modes with sufficiently different time scales. The obtained limit then implies the decoupling of the corresponding dynamics.

Index Terms-Limiting property, logarithmic norm, matrix exponential, time-scale separation.

\section{INTRODUCTION}

The subject of study in this paper is the matrix exponential

$$
\exp \left(\left[\begin{array}{cc}
A_{11}-K(\alpha) & A_{12} \\
A_{21} & A_{22}
\end{array}\right] t\right), \quad t>0
$$

Manuscript received October 15, 2012; revised June 07, 2013; accepted October 15, 2013. Date of publication October 24, 2013; date of current version March 20, 2014. This work was supported by the Swiss National Science Foundation (SNSF). Recommended by Associate Editor F. Blanchini.

S. Trimpe is with the Max Planck Institute for Intelligent Systems, 72076 Tübingen, Germany (e-mail: strimpe@tuebingen.mpg.de).

R. D'Andrea is with the Institute for Dynamic Systems and Control, ETH Zurich, 8092 Zurich, Switzerland (e-mail: rdandrea@ethz.ch).

Digital Object Identifier 10.1109/TAC.2013.2287112
The matrix function $K(\alpha)$ then represents the feedback gain parametrized by $\alpha$. The feedback system is depicted in Fig. 1. A more general multi-loop feedback system with additional reference inputs is considered in [1].

The matrix exponential (1) is a fundamental matrix (see e.g., [2]) of the feedback system (3)-(5). The limit (2) means that the dynamics of $x_{\mathrm{f}}(t)$ and $x_{\mathrm{s}}(t)$ are decoupled in the limit as $K(\alpha)$ grows large. In this context, we seek to determine what type of feedback yields a decoupling of the states in feedback from the remaining ones in the limit as the feedback gains become arbitrarily large. control systems with high-gain inner loops, since a decoupling of the states allows for a simplified system description and, hence, a simplified control design. The matrix result herein is applied in [1] to derive a time-scale separation algorithm for a cascaded control system with high-gain inner feedback loops. The algorithm yields a system description that includes the plant dynamics and the effect of the inner feedback loops. The obtained representation is useful, for example, for designing an outer-loop controller. This methodology is applied in the design of a cascaded feedback control system for an inverted pendulum in [1] and for a balancing cube (a multi-body 3-D inverted pendulum) in [3].

Related to the problem studied herein is the work by Campbell et al., [4], [5]. The authors consider the matrix exponential with its argument being a polynomial in $1 / \varepsilon$ and derive conditions for its convergence
This question is of interest, for example, when designing multi-loop 